\title{
MicroRNA-944 targets vascular endothelial growth factor to inhibit cell proliferation and invasion in osteosarcoma
}

\author{
TINGZHEN YAN ${ }^{1}$, SHIYONG ZHU ${ }^{2}$, JING ZHANG $^{3}$, GONGBIAO LU $^{1}$, \\ CHAOLIANG LV ${ }^{1}$, YANCHUN WEI ${ }^{1}$ and MINGHUA LUO ${ }^{4}$
}

\author{
${ }^{1}$ Department of Spine Surgery, Jining No. 1 People's Hospital, Jining, Shandong 272011; \\ ${ }^{2}$ Department of Spine Surgery, Lanling County People's Hospital of Shandong Province, Linyi, Shandong 277700; \\ ${ }^{3}$ Department of Pathology, Jining No. 1 People's Hospital, Jining, Shandong 272011; ${ }^{4}$ Department of Orthopedics, \\ The Second People's Hospital of Jingdezhen, Jingdezhen, Jiangxi 333000, P.R. China
}

Received February 2, 2018; Accepted July 12, 2018

DOI: $10.3892 / \mathrm{mmr} .2018 .9524$

\begin{abstract}
Emerging evidence has demonstrated that the dysregulation of microRNA (miRNA/miR) serves a crucial role in the tumorigenesis and tumor development of osteosarcoma (OS), primarily by affecting various pathological behaviors. Therefore, better knowledge of miRNA in OS may provide novel insights into the pathogenesis of OS, and may facilitate the development of promising therapeutics for patients with this disease. MiRNA-944 is frequently dysregulated in human cancers. However, the expression levels, functions and underlying mechanisms of miR-944 in OS remain largely elusive. In the present study, reverse transcription-quantitative polymerase chain reaction (RT-qPCR) was performed to detect miR-944 expression in OS tissues and cell lines. The regulatory influence of miR-944 in OS proliferation and invasion was determined with MTT and Transwell invasion assays. In addition, the mechanisms underlying the action of miR-944 in OS cells were elucidated through a series of experiments, including bioinformatics analysis, luciferase reporter assay, RT-qPCR and western blot analysis. Spearman's correlation analysis was utilized to examine the relationship between miR-944 and VEGF expression levels, and rescue experiments were applied to further verify whether VEGF mediates the role of miR-944 in OS. The results demonstrated that miR-944 was downregulated in cancer tissues and cell lines. Furthermore, exogenous miR-944 expression inhibited cell proliferation and invasion in OS in vitro. Vascular endothelial growth factor (VEGF) was identified as a direct target of miR-944 in OS and was overexpressed in cancer tissues. VEGF expression was inversely correlated with miR-944 expression in cancer
\end{abstract}

Correspondence to: Professor Minghua Luo, Department of Orthopedics, The Second People's Hospital of Jingdezhen, 3 Guangchang North Road, Jingdezhen, Jiangxi 333000, P.R. China E-mail: luominghua_jdz@126.com

Key words: osteosarcoma, microRNA-944, proliferation, invasion, vascular endothelial growth factor tissues. Rescue experiments demonstrated that overexpression of VEGF partially prevented the miR-944-induced inhibition of OS cell proliferation and invasion. These results suggested that miR-944 may serve a tumor suppressive role in OS by directly targeting VEGF. Therefore, miR-944 may be a promising target in the treatment of OS.

\section{Introduction}

Osteosarcoma (OS) arises from primitive bone-forming mesenchymal cells and is the most common type of primary bone neoplasm in children and adolescents (1). It is characterized by rapid progression and high metastatic potential and is associated with high mortality rates among adolescents and young adults $(2,3)$. Despite recent developments in combined treatment including surgery, chemotherapy, and radiotherapy, the overall therapeutic outcomes remain unsatisfactory with a median survival time of only 23 months (4). The unfavorable prognosis of patients with OS is largely due to the high rates of recurrence and local or distant metastasis of this malignancy (5). Genetic alterations, environmental ionizing radiation, and lesions are closely associated with OS development; nevertheless, the detailed mechanisms underlying OS pathogenesis remain largely unknown (6). Therefore, fully understanding the occurrence and development of OS is necessary to develop alternative therapeutic strategies that can improve the clinical management of this disease.

MicroRNA (miRNA/miR) are a group of noncoding RNA molecules 22-25 nucleotides in length (7). MiRNA have been identified as critical gene regulators (8). Given that $30 \%$ of human protein-coding genes may be regulated by miRNA (9), they may modulate thousands of genes and serve key roles in a wide range of biological processes, including cell proliferation, cycle, development, differentiation, and metabolism (10). Numerous studies have demonstrated that miRNA expression levels are dysregulated in almost all types of human malignancies, including OS (11), breast (12), lung (13), nasopharyngeal (14), and cervical cancers (15). Dysregulated miRNA have been implicated as oncogenes or tumor suppressors in OS formation and progression. For example, the upregulation of miR-93a promotes OS cell proliferation through the 
negative regulation of cyclin-dependent kinase inhibitor 1A (16). MiR-203 overexpression restricts cell proliferation and invasion, inducing apoptosis in OS through blockage of runt-related transcription factor 2 (17). Considering their important role in OS, the in-depth investigation of the molecular mechanisms underlying the roles of miRNA in cancer is important to facilitate the identification of novel therapeutic agents or targets for OS.

MiR-944 is frequently dysregulated in human cancers, such as endometrial (18), cervical (19) and breast cancer (20). However, the expression pattern, detailed functions and underlying mechanisms of miR-944 in OS remain largely elusive. Hence, the present study aimed to detect the expression pattern, role, and governing mechanisms of miR-944 in OS.

\section{Materials and methods}

Tissue collection. The present study was approved by the Ethics Committee of Jining No. 1 People's Hospital, and was performed according to the principles of the Declaration of Helsinki. Written informed consent was also provided from all patients enrolled in the present study. A total of 38 cancerous and adjacent tissues were obtained from OS patients (22 males, 16 females; age range, 16-53 years) in Jining No. 1 People's Hospital between March 2015 and February 2017. None of the patients had received radiotherapy or chemotherapy prior to surgical resection. All cancerous and adjacent tissues $(\sim 0.5-1 \mathrm{~cm}$ thick) were snap-frozen and were kept in liquid nitrogen until further use.

Cell culture. Four human OS cell lines (MG-63, SAOS-2, HOS, and U2OS) and the normal human osteoblast hFOB1.19 were acquired from American Type Culture Collection (Manassas, VA, USA). All cells were cultured in Dulbecco's modified Eagle's medium (DMEM) supplemented with $10 \%$ fetal bovine serum (FBS), $100 \mathrm{U} / \mathrm{ml}$ penicillin and $100 \mathrm{U} / \mathrm{ml}$ streptomycin (all from Gibco; Thermo Fisher Scientific, Inc., Waltham, MA, USA), and grown at $37^{\circ} \mathrm{C}$ in a humidified atmosphere containing $5 \% \mathrm{CO}_{2}$. Cells were harvested using $0.25 \%$ trypsin- $0.02 \%$ EDTA solution (Gibco; Thermo Fisher Scientific, Inc.) once cell density reached $\sim 80-90 \%$ confluency.

Transfection of oligonucleotides and plasmids. For transfection, cells were seeded into 24 -well plates at a density of $5 \times 10^{5}$ cells/well. MiR-944 mimics (Shanghai GenePharma Co., Ltd., Shanghai, China) were introduced into cells to increase miR-944 expression. MiRNA mimic negative control (miR-NC) was used as an internal control. The miR-944 mimics sequence was 5'-AAAUUAUUGUACAUCGGAUGA G-3' and the miR-NC sequence was 5'-UUCUCCGAACGU GUCACGUTT-3'. In order to increase VEGF expression, cells were transfected with the VEGF-overexpressing plasmid pcDNA3.1-VEGF (Integrated Biotech Solutions, Shanghai, China). An empty pcDNA3.1 plasmid was used as control. Cells were transfected with miR-944 mimics (100 pmol), miR-NC (100 pmol), pcDNA3.1-VEGF $(4.0 \mu \mathrm{g})$ or empty pcDNA3.1 plasmid $(4.0 \mu \mathrm{g})$ using Lipofectamine ${ }^{\circledR} 2000$ (Invitrogen; Thermo Fisher Scientific, Inc.) in accordance with the manufacturer's protocol. Transfected cells were incubated at $37^{\circ} \mathrm{C}$ in a humidified atmosphere containing $5 \% \mathrm{CO}_{2}$.
Reverse transcription-quantitative polymerase chain reaction (RT-qPCR) was performed to detect miR-944 expression at $48 \mathrm{~h}$ post-transfection. At $72 \mathrm{~h}$ post-transfection, western blot analysis was used to determine VEGF protein expression. MTT and Transwell invasion assays were conducted at 24 and $48 \mathrm{~h}$ post-transfection.

Reverse transcription-quantitative polymerase chain reaction $(R T-q P C R)$. Total RNA was extracted from tissues or all cultured cells using TRIzol ${ }^{\circledR}$ reagent (Invitrogen; Thermo Fisher Scientific, Inc.) according to the manufacturer's protocol. For analysis of miR-944 expression levels, complementary DNA (cDNA) was synthesized from total RNA with a TaqMan MicroRNA Reverse Transcription kit (Applied Biosystems; Thermo Fisher Scientific, Inc.). The temperature protocol for reverse transcription was as follows: $16^{\circ} \mathrm{C}$ for $30 \mathrm{~min}, 42^{\circ} \mathrm{C}$ for $30 \mathrm{~min}$ and $85^{\circ} \mathrm{C}$ for $5 \mathrm{~min}$. qPCR was carried out on an ABI 7900 thermocycler (Applied Biosystems; Thermo Fisher Scientific, Inc.), using a TaqMan MicroRNA PCR Kit (Applied Biosystems; Thermo Fisher Scientific, Inc.). The thermocycling conditions were as follows: $50^{\circ} \mathrm{C}$ for $2 \mathrm{~min}$, $95^{\circ} \mathrm{C}$ for $10 \mathrm{~min} ; 40$ cycles of denaturation at $95^{\circ} \mathrm{C}$ for $15 \mathrm{sec}$ and annealing/extension at $60^{\circ} \mathrm{C}$ for $60 \mathrm{sec}$. For determination of VEGF mRNA expression levels, total RNA was converted into cDNA with a PrimeScript RT Reagent Kit (Takara Bio, Dalian, China), and then cDNA was subjected to qPCR using a SYBR Premix Ex Taq ${ }^{\text {TM }}$ Kit (Takara Bio, Dalian, China). The temperature protocol for reverse transcription was as follows: $37^{\circ} \mathrm{C}$ for $15 \mathrm{~min}$ and $85^{\circ} \mathrm{C}$ for $5 \mathrm{sec}$. The cycling conditions for qPCR were as follows: $5 \mathrm{~min}$ at $95^{\circ} \mathrm{C}$, followed by 40 cycles of $95^{\circ} \mathrm{C}$ for $30 \mathrm{sec}$ and $65^{\circ} \mathrm{C}$ for $45 \mathrm{sec}$. U6 small nuclear (sn)RNA and $\beta$-actin were used as endogenous controls for miR-944 and VEGF mRNA, respectively. These genes were all of human origin and the primers designed were as follows: miR-944, 5'-GCGGCGGAAATTATTGTACATC-3' (forward) and 5'-ATCCAGTGCAGGGTCCGAGG-3' (reverse); U6 snRNA, 5'-CTCGCTTCGGCAGCACA-3' (forward) and 5'-AACGCT TCACGAATTTGCGT-3' (reverse); VEGF, 5'-CGTGTACGT TGGTGCCCGCT-3' (forward) and 5'-TCCTTCCTCCTG CCCGGCTC-3' (reverse); and $\beta$-actin, 5'-ACAGAGCCTCGC CTTTGCCGATC-3' (forward) and 5'-ATCCTTCTGACCCAT GCCCACCA-3' (reverse). All data were analyzed by the $2^{-\Delta \Delta C q}$ method (21).

MTT assay. Following $24 \mathrm{~h}$ of incubation at $37^{\circ} \mathrm{C}$ with $5 \%$ $\mathrm{CO}_{2}$, transfected cells $\left(\sim 1 \times 10^{6}\right.$ cells/well $)$ were harvested using $0.25 \%$ trypsin- $0.02 \%$ EDTA solution and prepared into single-cell suspensions. Subsequently, $3 \times 10^{3}$ cells were plated into 96-well plates with six parallel replicates and incubated at $37^{\circ} \mathrm{C}$ in a humidified atmosphere containing $5 \% \mathrm{CO}_{2}$. MTT assay was conducted at 24, 48 and $72 \mathrm{~h}$ following incubation. At every time point, $20 \mu \mathrm{l}$ of MTT solution $(5 \mathrm{mg} / \mathrm{ml}$; Sigma-Aldrich; Merck KGaA, Darmstadt, Germany) was added into each well. The supernatant was gently discarded following $4 \mathrm{~h}$ of incubation at $37^{\circ} \mathrm{C}$. Subsequently, cells were lysed in $150 \mu \mathrm{l}$ dimethyl sulfoxide (Sigma-Aldrich; Merck $\mathrm{KGaA}$ ) at $37^{\circ} \mathrm{C}$ for $10 \mathrm{~min}$. At every time point, a microplate reader was used to detect optical density values at a wavelength of $490 \mathrm{~nm}$ (Bio-Rad Laboratories, Inc., Hercules, CA, USA). 
Transwell invasion assay. At $48 \mathrm{~h}$ post-transfection, cells were collected and suspended in FBS-free DMEM. A total of $1 \times 10^{5}$ transfected cells (200 $\mu$ l) in FBS-free DMEM were seeded into the upper chamber of Transwell inserts that were precoated with Matrigel (BD Biosciences, San Jose, CA, USA). The lower chambers of Transwell inserts were filled with $500 \mu 1$ of DMEM supplemented with $10 \%$ FBS. Following $24 \mathrm{~h}$ of incubation, noninvasive cells located on the upper surface of the chamber were gently removed with a cotton swab. Invasive cells were fixed in $100 \%$ methanol at room temperature for $20 \mathrm{~min}$ and then stained with $0.5 \%$ crystal violet solution (Sigma-Aldrich; Merck $\mathrm{KGaA}$ ) at room temperature for $20 \mathrm{~min}$. Following washing three times with PBS (Gibco; Thermo Fisher Scientific, Inc.), the number of invasive cells in five randomly selected fields per insert was quantified under a Leica inverted microscope (Leica Microsystems GmbH, Wetzlar, Germany).

Bioinformatics analysis. The putative targets of miR-944 were predicted using TargetScan Human 7.1 (www.targetscan.org/vert_71/) and miRDB (mirdb.org/).

Luciferase reporter assay. Bioinformatics analysis predicted that VEGF is a potential target gene of miR-944. Luciferase reporter assays were performed to determine whether miR-944 can interact with the 3'-untranslated region (UTR) of VEGF. The wild-type (Wt) fragment of the VEGF 3'-UTR containing miR-944 binding sites and the mutant (Mut) fragment of VEGF 3'UTR lacking complementarity with miR-944 were chemically synthesized by Shanghai GenePharma Co., Ltd, and inserted into pMIR-GLOTM Luciferase vectors (Promega Corporation, Madison, WI, USA). Constructs containing the Wt or Mut fragments of VEGF 3'UTR were designated as pMIR-VEGF-3'-UTR Wt or pMIR-VEGF-3'-UTR Mut, respectively. To conduct the luciferase reporter assay, cells were plated into 24 -well plates at a density of $1.0 \times 10^{5}$ cells/well. Lipofectamine 2000 was used to cotransfect MG-63 and U2OS cells with pMIR-VEGF-3'-UTR Wt $(0.2 \mu \mathrm{g})$ or pMIR-VEGF-3'-UTR Mut $(0.2 \mu \mathrm{g})$ and miR-944 mimics $(50 \mathrm{pmol})$ or miR-NC (50 pmol). At $48 \mathrm{~h}$ post-transfection, cells were harvested, and luciferase activity was analyzed with a dual-Luciferase Reporter assay system (Promega Corporation) in accordance with the manufacturer's protocol. The activity of firefly luciferase was normalized to that of Renilla luciferase.

Western blot analysis. Proteins were isolated from tissues or cultured cells using cold radioimmunoprecipitation lysis buffer (Santa Cruz Biotechnology, Inc., Dallas, TX, USA), and total protein concentrations were evaluated using bicinchoninic assay kits (Beyotime Institute of Biotechnology, Haimen, China) in accordance with the manufacturer's protocol. Equal amounts of protein (30 $\mu \mathrm{g} /$ lane) were separated through $10 \%$ SDS-PAGE and then transferred onto polyvinylidene membranes (Sigma-Aldrich; Merck KGaA). Following blocking at room temperature for $2 \mathrm{~h}$ in $5 \%$ fat-free milk in Tris-buffered saline containing $0.1 \%$ Tween-20 (TBST), the membranes were incubated overnight at $4^{\circ} \mathrm{C}$ with primary antibodies against VEGF (cat. no. ab1316; 1:1,000 dilution) or GAPDH (cat. no. ab125247; 1:1,000 dilution; both from Abcam, Cambridge, UK). Following three washes with TBST, the membranes were incubated with goat anti-mouse horseradish peroxidase-conjugated secondary antibodies (cat. no. ab205719; 1:5,000 dilution; Abcam) at room temperature for $2 \mathrm{~h}$. Protein signals were visualized by enhanced chemiluminescence Protein Detection kit (Pierce; Thermo Fisher Scientific, Inc.). The densities of protein signals were analyzed with ImageJ software (version 1.48; National Institutes of Health, Bethesda, MD, USA).

Statistical analysis. All results were expressed as the mean \pm standard deviation from at least three independent experiments. All statistical tests were conducted using SPSS software (version 18.0; SPSS, Inc., Chicago, IL, USA). Differences between two groups were analyzed with paired Student's t-test, and one-way analysis of variance was performed for multiple groups with Student Newman Keuls as a post hoc test. The correlation between miR-944 and VEGF mRNA expression levels was examined using Spearman's correlation analysis. $\mathrm{P}<0.05$ was considered to indicate a statistically significant difference.

\section{Results}

MiR-944 expression is downregulated in cancer tissues and cell lines. MiR-944 expression in 38 pairs of OS cancer and adjacent tissues was investigated by RT-qPCR. As illustrated in Fig. 1A, miR-944 expression in cancer tissues was decreased compared with that in adjacent tissues $(\mathrm{P}<0.05)$. MiR-944 expression levels were also investigated in four OS cell lines and the normal human osteoblast cell line hFOB1.19. Results demonstrated that miR-944 expression was downregulated in all four OS cell lines compared with in hFOB1.19 $(\mathrm{P}<0.05$; Fig. 1B). These results suggested that miR-944 is downregulated in OS, and this downregulation may be associated with the development of OS.

MiR-944 overexpression inhibits OS cell proliferation and invasion. Given that miR-944 was decreased in OS, it was hypothesized that miR-944 may have a tumor-suppressive role in OS. To confirm this hypothesis, MG-63 and U2OS cells were selected, which exhibited lower miR-944 expression than the other cell lines, for further experiments. MG-63 and U2OS cells were transfected with miR-944 mimics or miR-NC. RT-qPCR analysis demonstrated that miR-944 expression levels in MG-63 and U2OS cells transfected with miR-944 mimics were increased compared with that in MG-63 and U2OS cells transfected with miR-NC ( $\mathrm{P}<0.05$; Fig. 2A). MTT assay results revealed that the upregulation of miR-944 expression levels significantly reduced the proliferation of MG-63 and U2OS cells $(\mathrm{P}<0.05$; Fig. 2B). Furthermore, Transwell invasion assays indicated that miR-944 overexpression decreased the invasive capabilities of MG-63 and U2OS cells $(\mathrm{P}<0.05$; Fig. 2C and D). These results suggested that miR-944 may act as a tumor suppressor in OS in vitro.

VEGF is a direct target of miR-944 in OS. Bioinformatics analysis was performed to predict the putative targets of miR-944 and to delineate the mechanism responsible for the tumor-suppressing role of miR-944 in OS. As demonstrated in Fig. 3A, the 3'-UTR of VEGF contains a highly conserved binding site for miR-944. Thus, VEGF was selected for investigation to further verify that it serves a crucial role in 

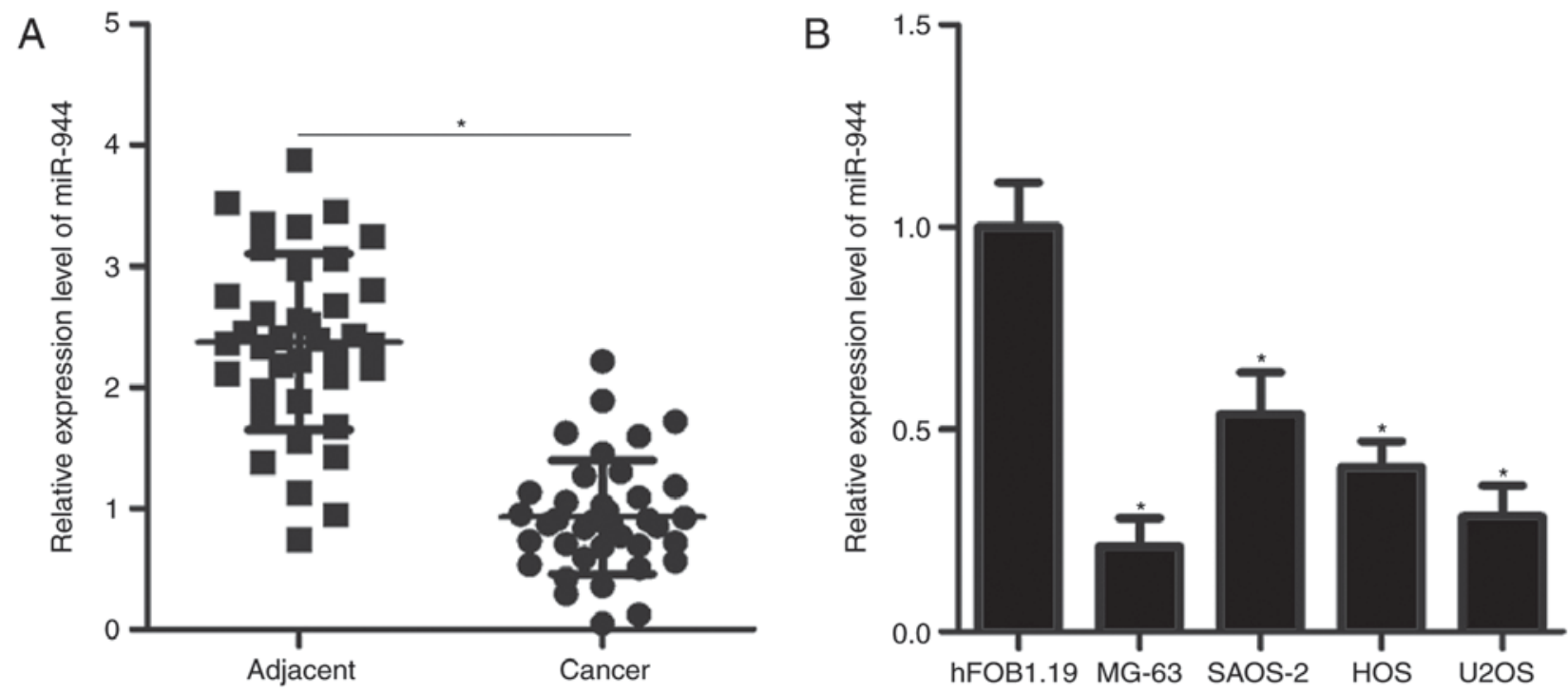

Figure 1. miR-944 expression is downregulated in cancer tissues and cell lines. (A) MiR-944 expression levels in 38 pairs of cancer and adjacent tissues were detected by RT-qPCR. "P<0.05 vs. adjacent tissues. (B) Relative miR-944 expression levels in four OS cell lines (MG-63, SAOS-2, HOS, and U2OS) and the normal human osteoblast cell line hFOB1.19 were investigated through RT-qPCR analysis. ${ }^{\text {P }}<0.05$ vs. hFOB1.19. miR, microRNA; OS, osteosarcoma; RT-qPCR, reverse transcription-quantitative polymerase chain reaction.
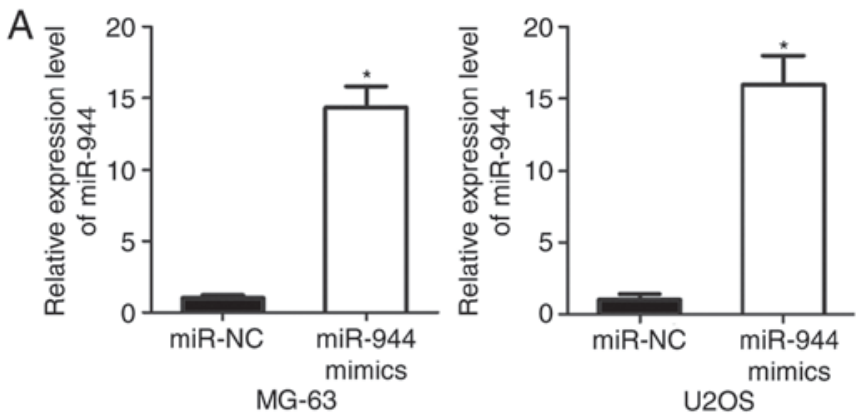

U2OS

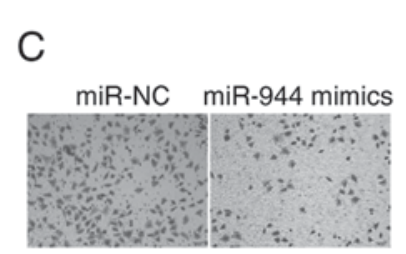

MG-63

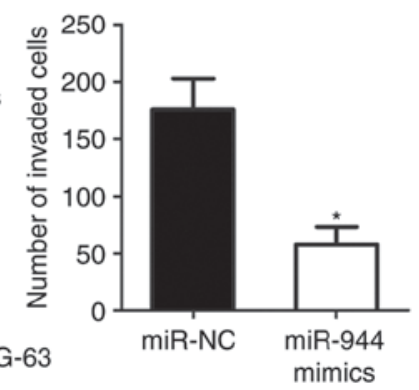

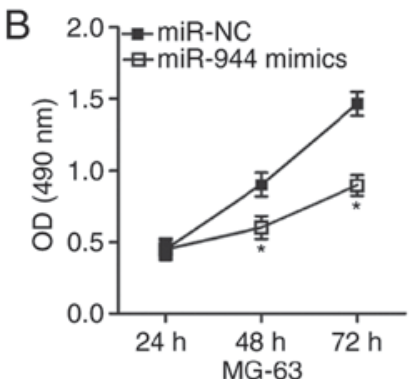
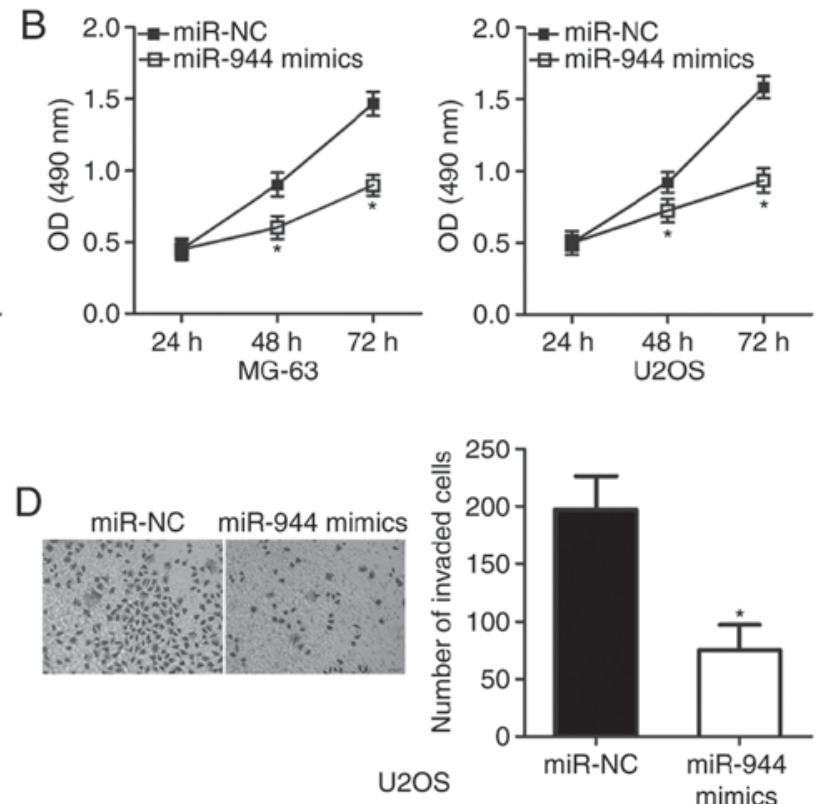

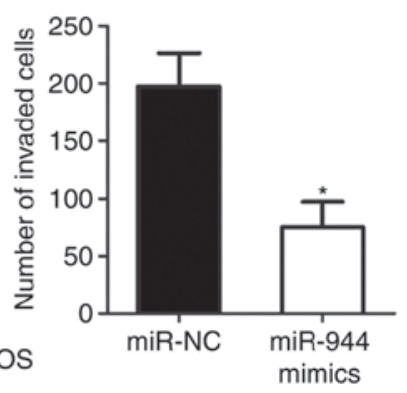

Figure 2. Overexpression of miR-944 suppresses the proliferation and invasion of MG-63 and U2OS cells. (A) MG-63 and U2OS cells were transfected with miR-944 mimics or miR-NC. MiR-944 expression was quantified through reverse transcription-quantitative polymerase chain reaction. (B) Transfected cells were inoculated into 96-well plates, and cell proliferation was evaluated through MTT assay. OD was measured at $490 \mathrm{~nm}$. Transwell invasion assay was applied to determine the invasive capacity of (C) MG-63 and (D) U2OS cells transfected with miR-944 mimics or miR-NC. "P<0.05 vs. miR-NC. miR, microRNA; NC, negative control; OS, osteosarcoma; OD, optical density.

OS onset and development (22-25). Subsequently, whether the putative binding site has functional relevance was investigated by luciferase reporter assays. As speculated, overexpression of miR-944 decreased the luciferase activity of the pMIR-VEGF-3'-UTR Wt group $(\mathrm{P}<0.05)$ but failed to affect that of the pMIR-VEGF-3'-UTR Mut group (Fig. 3B). This result demonstrated that miR-944 could directly interact with the 3'-UTR of VEGF. In addition, RT-qPCR and western blot analysis results indicated that the overexpression of miR-944 significantly suppressed VEGF mRNA (P<0.05; Fig. $3 C)$ and protein $(\mathrm{P}<0.05$; Fig. 3D) expression levels in MG-63 and U2OS cells.

VEGF is upregulated in cancer tissues, and its expression is negatively correlated with miR-944 expression. To further clarify the association between miR-944 and VEGF in OS, VEGF mRNA expression was investigated in 38 pairs of cancer tissues and adjacent tissues. RT-qPCR analysis revealed that the mRNA level of VEGF was increased in cancerous compared with adjacent tissues $(\mathrm{P}<0.05$; Fig. 4A). In addition, 
A

$\begin{array}{ll}\text { VEGF-3'-UTR wild-type } & 5^{\prime} \\ \text { hsa-miR-944 } & 3^{\prime} \\ \text { VEGF-3'-UTR mutant } & 5^{\prime}\end{array}$

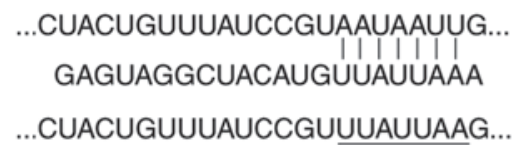

B
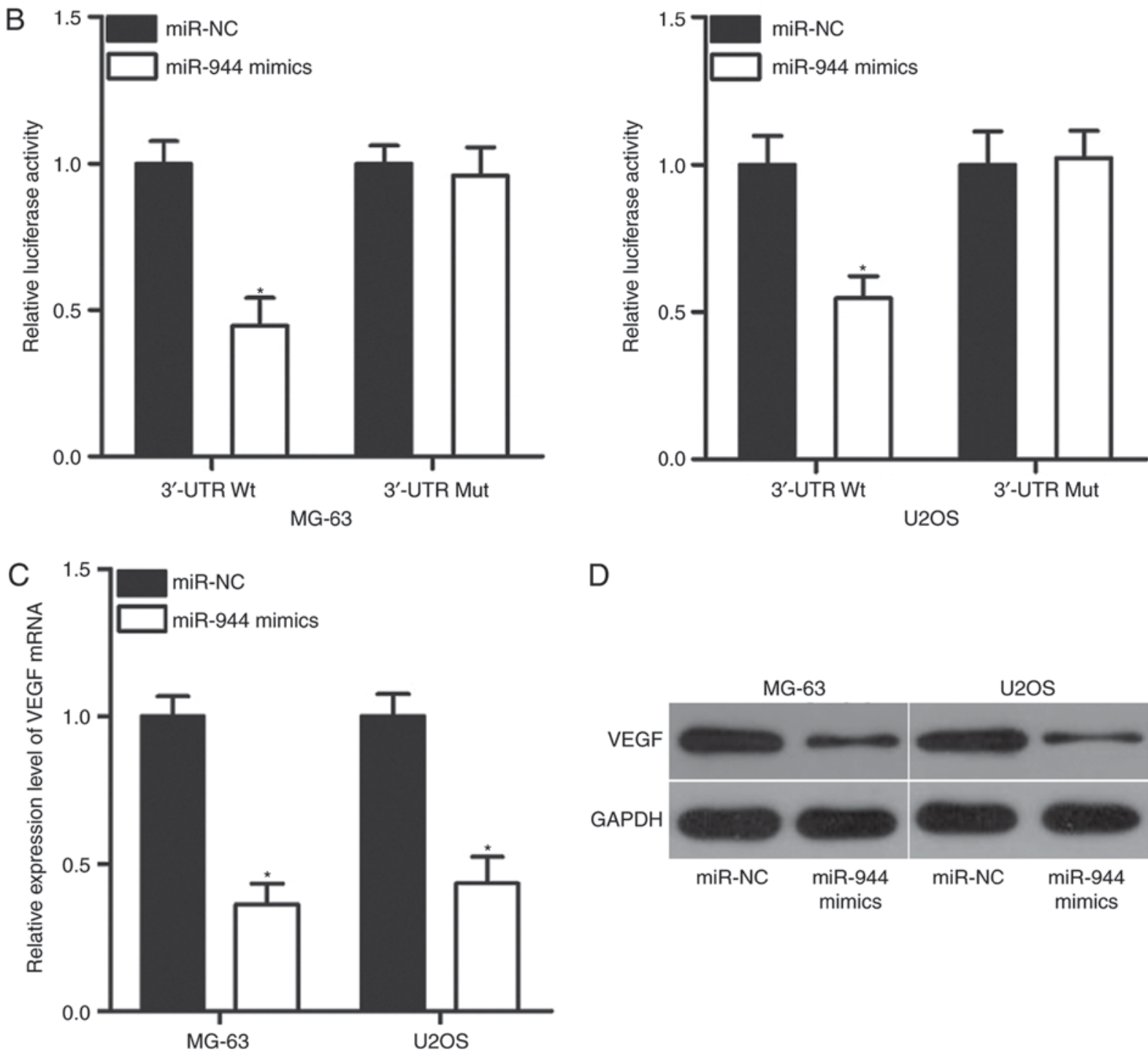

D

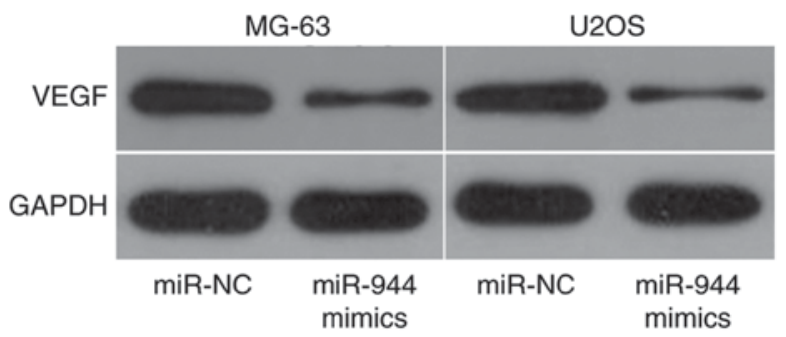

Figure 3. VEGF is a direct target gene of miR-944 in OS. (A) Putative wild-type (Wt) and mutant (Mut) target sequences for miR-944 in the 3'-UTR of VEGF. (B) Luciferase reporter assay results for MG-63 and U2OS cells cotransfected with miR-944 mimics or miR-NC and Wt or Mut luciferase reporter plasmid. (C) Reverse transcription-quantitative polymerase chain reaction and (D) western blot analysis were performed to quantify VEGF mRNA and protein expression levels, respectively, in MG-63 and U2OS cells transfected with miR-944 mimics or miR-NC. "P<0.05 vs. miR-NC. miR, microRNA; Mut, mutated; NC, negative control; OS, osteosarcoma; UTR, untranslated region; VEGF, vascular endothelial growth factor; Wt, wild type.

the results of western blot analysis demonstrated that VEGF protein was overexpressed in cancer tissues compared with adjacent tissues (Fig. 4B). Furthermore, Spearman's correlation analysis revealed that miR-944 and VEGF mRNA levels were inversely correlated in cancer tissues $(\mathrm{r}=-0.5692, \mathrm{P}=0.0002$; Fig. 4C).

Overexpression of VEGF expression levels abolishes the inhibitory effects of miR-944 overexpression in OS cells. To further verify whether VEGF mediates the role of miR-944 in OS, MG-63 and U2OS cells were cotransfected with miR-944 mimics and empty pcDNA3.1 plasmid or pcDNA3.1-VEGF. The miR-944-mediated downregulation of VEGF protein expression levels was almost reversed in MG-63 and U2OS cells cotransfected with pcDNA3.1-VEGF ( $\mathrm{P}<0.05$; Fig. 5A). Additionally, overexpression of VEGF expression counteracted the suppressive effects of miR-944 overexpression on proliferation $(\mathrm{P}<0.05$; Fig. $5 \mathrm{~B})$ and invasion $(\mathrm{P}<0.05$; Fig. $5 \mathrm{C}$ and $\mathrm{D})$ of MG-63 and U2OS cells. These results suggested that miR-944 exhibits a tumor suppressive role in OS by inhibiting VEGF expression in vitro.

\section{Discussion}

Emerging evidence has demonstrated that the dysregulation of miRNA serves crucial roles in the tumorigenesis and tumor 
A

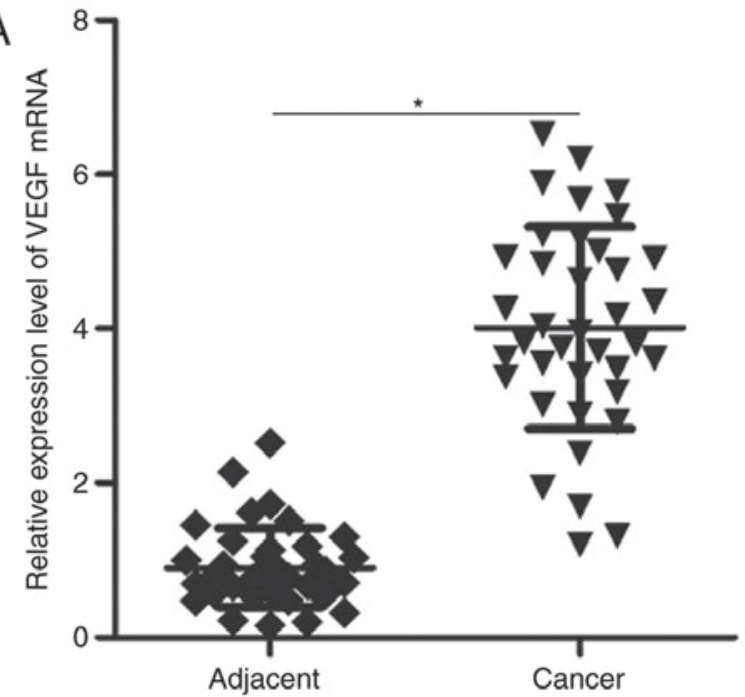

B

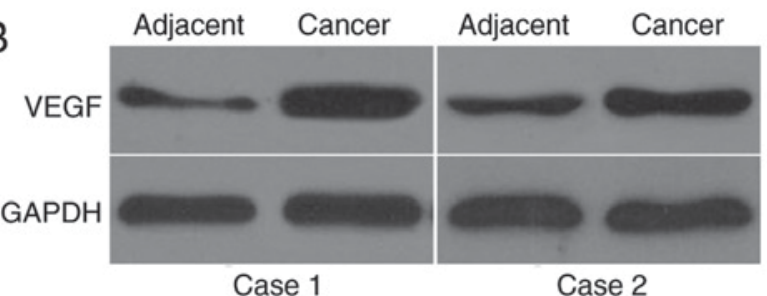

Case 1

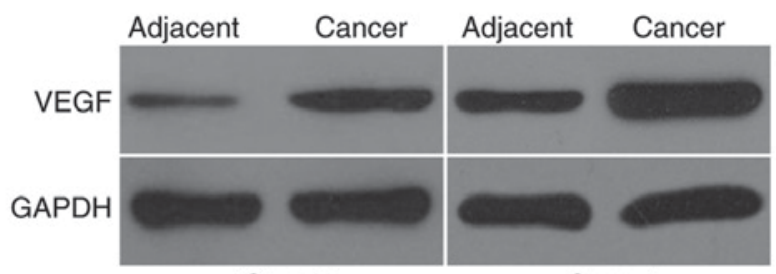

Case 3

Case 4

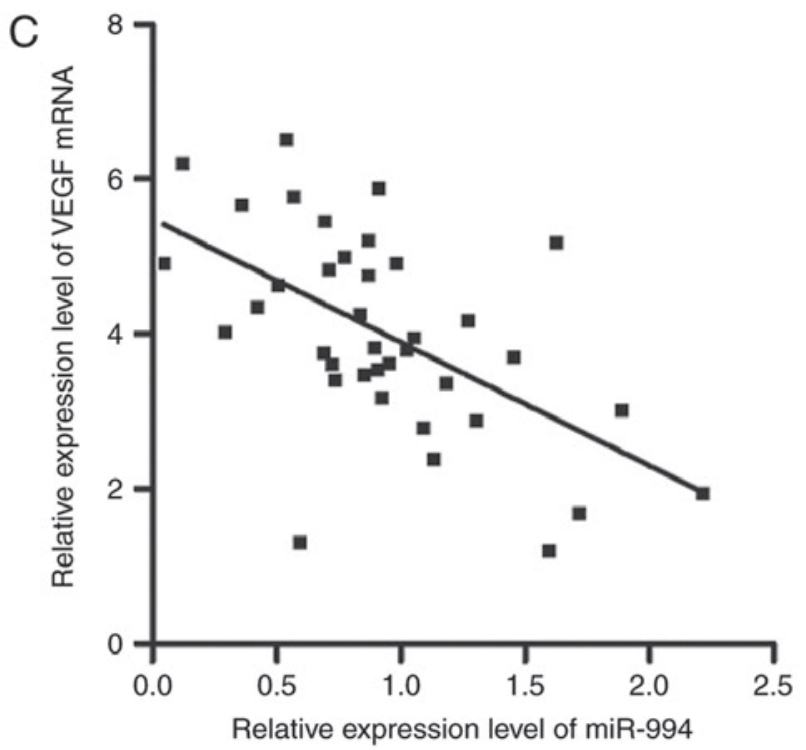

Figure 4. VEGF is overexpressed in cancer tissues, and expression levels are inversely correlated with miR-944 expression levels. VEGF (A) mRNA and (B) protein expression levels were detected in cancerous and adjacent tissues. (C) Spearman's correlation analysis was performed to assess the correlation between VEGF and miR-944 expression levels in cancer tissues. " $\mathrm{P}<0.05$ vs. adjacent tissues; correlation analysis, $\mathrm{r}=-0.5692, \mathrm{P}=0.0002$. miR, microRNA; OS, osteosarcoma; VEGF, vascular endothelial growth factor.

development of OS, mainly by affecting various pathological behaviors $(26,27)$. Therefore, better knowledge of miRNAs in OS may provide novel insights into the pathogenesis of OS, and may help the development of promising therapeutic targets for treating patients with this disease. The present study demonstrated that miR-944 was significantly downregulated in cancer tissues and cell lines. Further investigation indicated that overexpression of miR-944 expression inhibited cell proliferation and invasion in OS cell lines. Additionally, VEGF was validated as a direct target of miR-944 in OS. VEGF was upregulated in cancer tissues and this upregulation was inversely correlated with miR-944 expression. Furthermore, overexpression of VEGF reversed the tumour-suppressive effects of miR-944 on cell proliferation and invasion in OS in vitro. These results demonstrated that miR-944 can exert tumor suppressive-like behavior in OS by directly targeting VEGF. Hence, miR-944 may be identified as an effective target for the treatment of patients with OS, but further investigation is required.
Previous studies have demonstrated that miR-944 is aberrantly expressed in several malignant phenotypes of human cancer (18-20). For example, miR-944 is upregulated in endometrial cancer tissues and cell lines. Its increased level is significantly correlated with pathological classification by the International Federation of Gynaecology and Obstetrics Stages of Endometrial Cancer (18). He et al (18) demonstrated that the overexpression of miR-944 increases endometrial cancer cell proliferation, cell cycle progression and reduces apoptosis in vitro. Conversely, knockdown of miR-944 suppresses the tumour growth of endometrial cancer in vivo, while Kaplan-Meier analysis indicates that high miR-944 expression levels are associated with poor overall survival of patients with endometrial cancer (18). Furthermore, miR-944 has been demonstrated to be overexpressed in cervical cancer, and Xie et al (19) demonstrated that downregulating miR-944 attenuates cell proliferation and motility in cervical cancer, and breast cancer. In addition, He et al (20) revealed 

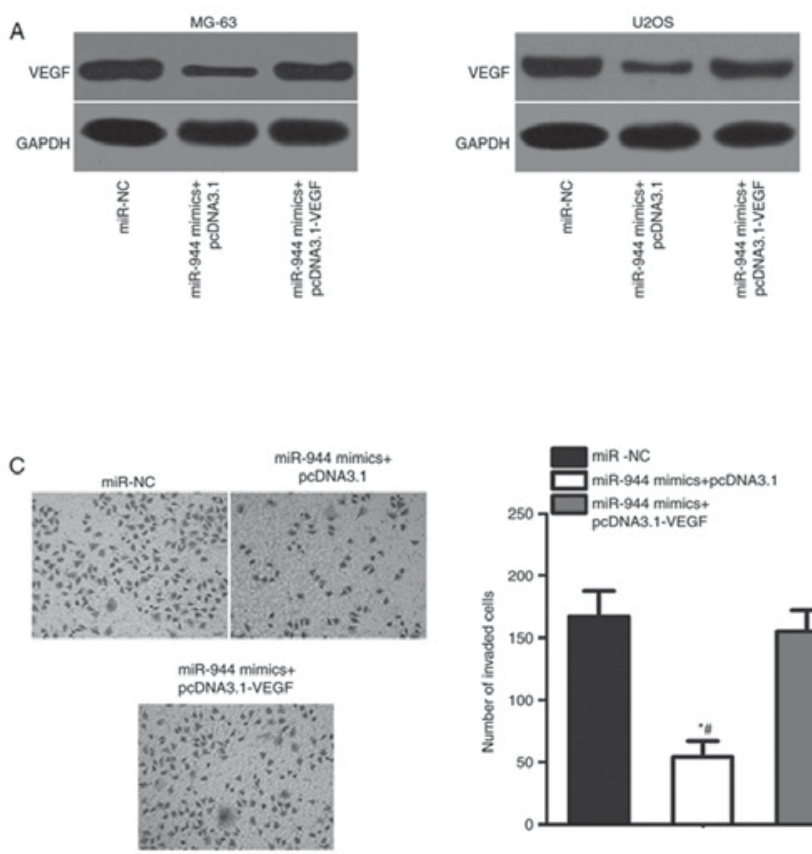
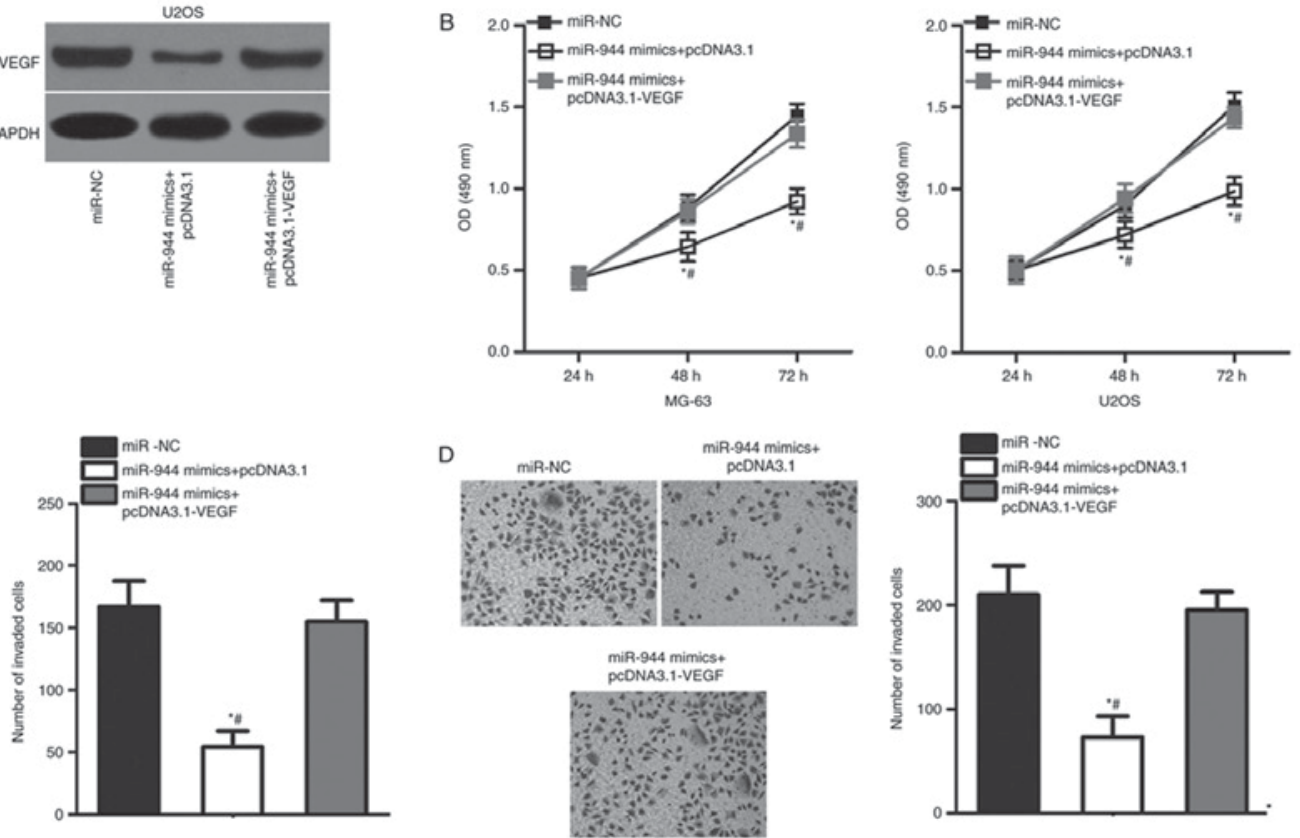

MG-63

U20s

Figure 5. Overexpression of VEGF abolishes the inhibitory effects of miR-944 overexpression on the proliferative and invasive abilities of MG-63 and U2OS cells. (A) MG-63 and U2OS cells were transfected with pcDNA3.1 or pcDNA3.1-VEGF in the presence of miR-944 mimics. (B) Cell proliferation in both cells lines was assessed by MTT and invasion in (C) MG-63 and in (D) U2OS cells was assessed by Transwell invasion assay. $\mathrm{P}<0.05 \mathrm{~b}$ vs. miR-NC. "P<0.05 vs. miR-944 mimics+pcDNA3.1-VEGF. miR, microRNA; NC, negative control; OS, osteosarcoma; VEGF, vascular endothelial growth factor.

that ectopic expression of miR-944 promotes cell growth and metastasis in breast cancer. Additionally, the same study demonstrated that downregulation of miR-944 increased the cytotoxicity of cisplatin in cisplatin-resistant breast cancer cells. However, miR-944 is notably downregulated in colorectal cancer tissues and cell lines. Low expression level of miR-944 is associated with malignant clinical parameters and poor prognosis of patients with colorectal cancer (28). In the same study, miR-944 was identified as a tumour suppressor in colorectal cancer as it inhibits cell migration and invasion. Downregulation of miR-944 has also been observed in gastric cancer (29) where it has been reported that resumed expression of miR-944 decreases metastasis and epithelial-mesenchymal transition, and in non-small cell lung cancer, where miR-944 overexpression inhibits cell proliferation (30). These findings suggest that the functional roles of miR-944 in human malignancies have tissue specificity and miR-944 should be investigated as a potential therapeutic target for the aforementioned cancer types, but this needs to be investigated further.

Identification of the direct targets of miR-944 may provide a molecular basis for investigation of the regulatory mechanisms of miR-944. Several targets of miR-944 have been validated and include cell adhesion molecule 2 (18) in endometrial cancer, C2 and WW domain containing E3 ubiquitin protein ligase 2 (19) and S100P binding protein (19) in cervical cancer, B cell lymphoma 2 interacting protein 3 (20) in breast cancer, metastasis associated in colon cancer 1 (28) in colorectal cancer, and Eph receptor A7 (30) in non-small cell lung cancer. VEGF, also known as VEGFA, was demonstrated to be a direct target gene of miR-944 in OS, in the present study. It is aberrantly overexpressed in a variety of human malignancies, such as gastric cancer (31), thyroid cancer (32), bladder cancer (33), and colorectal cancer (34). Overexpression of VEGF has also been reported in OS, and this upregulation is strongly correlated with Enneking stage and distant metastasis (22). OS patients with high VEGF expression levels exhibit an unfavorable prognosis compared with patients with low VEGF levels (23). VEGF serves an important role in OS initiation and progression via regulating cell proliferation, apoptosis, migration, invasion, metastasis, and angiogenesis $(24,25)$. Hence, knocking down VEGF expression levels may be an attractive therapeutic approach in the therapy of patients with OS.

In conclusion, the present study demonstrated that miR-944 was significantly downregulated in cancer tissues and cell lines. Upregulating miR-944 inhibited cellular proliferation and invasion in OS cells by directly targeting VEGF. These results suggest a theoretical basis for the application of miR-944 in treating patients with OS. However, several limitations of the present study should be recognised. The number of cases in the present study was small. In addition, normal bone tissues were not used as a control. Furthermore, the effects of miR-944 on an OS animal model were not studied. Therefore, future in vitro and in vivo experiments need to be performed in order to validate the present findings.

\section{Acknowledgements}

Not applicable.

\section{Funding}

No funding was received. 


\section{Availability of data and materials}

The analyzed data sets generated during the present study are available from the corresponding author on reasonable request.

\section{Authors' contributions}

ML and TY designed this research, wrote and revised the manuscript. TY, SZ and JZ performed RT-qPCR, western blot analysis and MTT assay. GL, CL and YW conducted the Transwell invasion and luciferase reporter assay. All authors read and approved the final manuscript.

\section{Ethics approval and consent to participate}

The present study was approved by the Ethics Committee of Jining No. 1 People's Hospital, and was performed according to the principles of the Declaration of Helsinki. Written informed consent was also provided from all patients enrolled in the present study.

\section{Patient consent for publication}

Written informed consent was provided.

\section{Competing interests}

The authors declare that they have no competing interests.

\section{References}

1. Siegel RL, Miller KD and Jemal A: Cancer statistics, 2016. CA Cancer J Clin 66: 7-30, 2016.

2. Kansara M, Teng MW, Smyth MJ and Thomas DM: Translational biology of osteosarcoma. Nat Rev Cancer 14: 722-735, 2014.

3. Gianferante DM, Mirabello L and Savage SA: Germline and somatic genetics of osteosarcoma-connecting aetiology, biology and therapy. Nat Rev Endocrinol 13: 480-491, 2017.

4. Fagioli F, Aglietta M, Tienghi A, Ferrari S, Brach del Prever A Vassallo E, Palmero A, Biasin E, Bacci G, Picci P and Madon E: High-dose chemotherapy in the treatment of relapsed osteosarcoma: An Italian sarcoma group study. J Clin Oncol 20: 2150-2156, 2002.

5. Wada T, Isu K, Takeda N, Usui M, Ishii S and Yamawaki S: A preliminary report of neoadjuvant chemotherapy NSH-7 study in osteosarcoma: Preoperative salvage chemotherapy based on clinical tumor response and the use of granulocyte colony-stimulating factor. Oncology 53: 221-227, 1996.

6. Tan ML, Choong PF and Dass CR: Osteosarcoma: Conventional treatment vs. gene therapy. Cancer Biol Ther 8: 106-117, 2009.

7. Bartel DP: MicroRNAs: Genomics, biogenesis, mechanism, and function. Cell 116: 281-297, 2004.

8. Calin GA and Croce CM: MicroRNA signatures in human cancers. Nat Rev Cancer 6: 857-866, 2006.

9. Lewis BP, Burge CB and Bartel DP: Conserved seed pairing, often flanked by adenosines, indicates that thousands of human genes are microRNA targets. Cell 120: 15-20, 2005.

10. Lin S and Gregory RI: MicroRNA biogenesis pathways in cancer. Nat Rev Cancer 15: 321-333, 2015.

11. Ghosh T, Varshney A, Kumar P, Kaur M, Kumar V, Shekhar R, Devi R, Priyanka P, Khan MM and Saxena S: MicroRNA-874-mediated inhibition of the major $\mathrm{G}_{1} / \mathrm{S}$ phase cyclin, CCNE1, is lost in osteosarcomas. J Biol Chem 292: 21264-21281, 2017.

12. Li D, Wang H, Song $\mathrm{H}, \mathrm{Xu} \mathrm{H}$, Zhao B, Wu C, Hu J, Wu T, Xie D, Zhao J, et al: The microRNAs miR-200b-3p and miR-429-5p target the LIMK1/CFL1 pathway to inhibit growth and motility of breast cancer cells. Oncotarget 8: 85276-85289, 2017.
13. Huang RS, Zheng YL, Zhao J and Chun X: microRNA-381 suppresses the growth and increases cisplatin sensitivity in non-small cell lung cancer cells through inhibition of nuclear factor-kB signaling. Biomed Pharmacother 98: 538-544, 2018.

14. Zhao F, Pu Y, Qian L, Zang C, Tao Z and Gao J: MiR-20a-5p promotes radio-resistance by targeting NPAS2 in nasopharyngeal cancer cells. Oncotarget 8: 105873-105881, 2017.

15. Zhao Y, Liu X and Lu YX: MicroRNA-143 regulates the proliferation and apoptosis of cervical cancer cells by targeting HIF-1 $\alpha$. Eur Rev Med Pharmacol Sci 21: 5580-5586, 2017.

16. He Y and Yu B: MicroRNA-93 promotes cell proliferation by directly targeting P21 in osteosarcoma cells. Exp Ther Med 13: 2003-2011, 2017.

17. Lin W, Zhu X, Yang S, Chen X, Wang L, Huang Z, Ding Y, Huang L and Lv C: MicroRNA-203 inhibits proliferation and invasion, and promotes apoptosis of osteosarcoma cells by targeting Runt-related transcription factor 2 . Biomed Pharmacother 91: 1075-1084, 2017.

18. He Z, Xu H, Meng Y and Kuang Y: miR-944 acts as a prognostic marker and promotes the tumor progression in endometrial cancer. Biomed Pharmacother 88: 902-910, 2017.

19. Xie H, Lee L, Scicluna P, Kavak E, Larsson C, Sandberg R and Lui WO: Novel functions and targets of miR-944 in human cervical cancer cells. Int J Cancer 136: E230-E241, 2015.

20. He H, Tian W, Chen $\mathrm{H}$ and Jiang K: MiR-944 functions as a novel oncogene and regulates the chemoresistance in breast cancer. Tumour Biol 37: 1599-1607, 2016.

21. Livak KJ and Schmittgen TD: Analysis of relative gene expression data using real-time quantitative PCR and the 2(-Delta Delta C(T)) method. Methods 25: 402-408, 2001.

22. Liu Y, Zhang F, Zhang Z, Wang D, Cui B, Zeng F, Huang L, Zhang Q and Sun Q: High expression levels of Cyr61 and VEGF are associated with poor prognosis in osteosarcoma. Pathol Res Pract 213: 895-899, 2017.

23. Yu XW, Wu TY, Yi X, Ren WP, Zhou ZB, Sun YQ and Zhang CQ: Prognostic significance of VEGF expression in osteosarcoma: A meta-analysis. Tumour Biol 35: 155-160, 2014.

24. Mei J, Gao Y, Zhang L, Cai X, Qian Z, Huang H and Huang W: VEGF-siRNA silencing induces apoptosis, inhibits proliferation and suppresses vasculogenic mimicry in osteosarcoma in vitro. Exp Oncol 30: 29-34, 2008.

25. Peng N, Gao S, Guo X, Wang G, Cheng C, Li M and Liu K: Silencing of VEGF inhibits human osteosarcoma angiogenesis and promotes cell apoptosis via VEGF/PI3K/AKT signaling pathway. Am J Transl Res 8: 1005-1015, 2016.

26. Kushlinskii NE, Fridman MV and Braga EA: Molecular mechanisms and microRNAs in osteosarcoma pathogenesis. Biochemistry (Mosc) 81: 315-328, 2016.

27. Sampson VB, Yoo S, Kumar A, Vetter NS and Kolb EA: MicroRNAs and potential targets in osteosarcoma: Review. Front Pediatr 3: 69, 2015.

28. Wen L, Li Y, Jiang Z, Zhang Y, Yang B and Han F: miR-944 inhibits cell migration and invasion by targeting MACC1 in colorectal cancer. Oncol Rep 37: 3415-3422, 2017.

29. Pan T, Chen W, Yuan X, Shen J, Qin C and Wang L: miR-944 inhibits metastasis of gastric cancer by preventing the epithelial-mesenchymal transition via MACC1/Met/AKT signaling. FEBS Open Bio 7: 905-914, 2017.

30. Liu M, Zhou K and Cao Y: MicroRNA-944 affects cell growth by targeting EPHA7 in non-small cell lung cancer. Int J Mol Sci 17: pii: E1493, 2016

31. Ji YN, Wang Q, Li Y and Wang Z: Prognostic value of vascular endothelial growth factor A expression in gastric cancer: A meta-analysis. Tumour Biol 35: 2787-2793, 2014.

32. Tian XF, Zhang XW, Chen RX, Wang JW and Zhang D: Clinical significance of expression of VEGF and bFGF in thyroid carcinoma. Zhonghua Wai Ke Za Zhi 42: 864-866, 2004 (In Chinese).

33. Huang YJ, Qi WX, He AN, Sun YJ, Shen Z and Yao Y: Prognostic value of tissue vascular endothelial growth factor expression in bladder cancer: A meta-analysis. Asian Pac J Cancer Prev 14: 645-649, 2013.

34. Wen L, Wang R, Lu X and You C: Expression and clinical significance of vascular endothelial growth factor and fms-related tyrosine kinase 1 in colorectal cancer. Oncol Lett 9: 2414-2418, 2015. 